\title{
Generalized Reduced Rank Tests using the Singular Value Decomposition*
}

\author{
Frank Kleibergen ${ }^{\dagger}$ \\ Department of Quantitative Economics \\ University of Amsterdam
}

\author{
Richard Paap \\ Econometric Institute \\ Erasmus University Rotterdam
}

February 26, 2004

\begin{abstract}
We propose a novel statistic to test the rank of a matrix. The rank statistic overcomes deficiencies of existing rank statistics, like: necessity of a Kronecker covariance matrix for the canonical correlation rank statistic of Anderson (1951), sensitivity to the ordering of the variables for the LDU rank statistic of Cragg and Donald (1996) and Gill and Lewbel (1992), a limiting distribution that is not a standard chi-squared distribution for the rank statistic of Robin and Smith (2000) and usage of numerical optimization for the objective function statistic of Cragg and Donald (1997). The new rank statistic consists of a quadratic form of a (orthogonal) transformation of the smallest singular values of a unrestricted estimate of the matrix of interest. The quadratic form is taken with respect to the inverse of a unrestricted covariance matrix that can be estimated using a heteroscedasticity autocorrelation consistent estimator. The rank statistic has a standard $\chi^{2}$ limiting distribution. In case of a Kronecker covariance matrix, the rank statistic simplifies to the canonical correlation rank statistic. In the non-stationary cointegration case, the limiting distribution of the rank statistic is identical to that of the Johansen trace statistic. We apply the rank statistic to test for the rank of a matrix that governs the identification of the parameters in the stochastic discount factor model of Jagannathan and Wang (1996). The rank statistic shows that non-identification of the parameters can not be rejected. We further use the stochastic discount factor model to illustrate the validity of the limiting distribution and to conduct a power comparison.
\end{abstract}

Key words: stochastic discount factor model, cointegration, GMM.

JEL codes: C12, C13, C30

\footnotetext{
*The first authors' research documented in this article has been funded by the NWO Vernieuwingsimpuls research grant "Empirical Comparison of Economic Models".

${ }^{\dagger}$ Department of Quantitative Economics, University of Amsterdam, Roetersstraat 11, 1018 WB Amsterdam, The Netherlands, e-mail: kleiberg@fee.uva.nl, homepage: http://www.fee.uva.nl/ke/kleibergen.htm

$\ddagger$ Econometric Institute (H11-25), Erasmus University Rotterdam, P.O. Box 1738, NL-3000 DR Rotterdam, The Netherlands, e-mail: paap@few.eur.nl, homepage: http://www.few.eur.nl/few/people/paap
} 


\section{Introduction}

Tests of many economic hypotheses boil down to testing the rank of a matrix, see for example Lewbel (1991). Also the identification of parameters in econometric models often depends on the rank of a matrix. Therefore, since the early stages of the development of econometric methodology a literature focusing on rank tests has developed. An early contribution to this line of work is Anderson (1951). Anderson (1951) proposes a likelihood ratio rank statistic in a multivariate regression model based on canonical correlations. This canonical correlation rank statistic has a $\chi^{2}$ limiting distribution when the covariance matrix of the unrestricted matrix estimator has a Kronecker structure. A Kronecker covariance matrix is however not always valid. For example, it does not apply if one opts for the use of heteroscedasticity autocorrelation consistent covariance (HACC) matrix estimators, see for example Andrews (1991), Newey and West (1987), West (1997) and White (1980). Alternative approaches to test the rank of a matrix that overcome this deficit have therefore been proposed.

Gill and Lewbel (1992) and Cragg and Donald (1996) use a LDU decomposition (see for example Golub and van Loan (1989)) of the unrestricted matrix to construct a rank statistic. They use a $($ root- $N)$ consistent estimator of the unrestricted matrix, which does not need to have a Kronecker covariance matrix. A disadvantage of their approach is that it can be sensitive to the ordering of the variables in the model. To overcome this sensitivity, the estimator of the unrestricted matrix is permuted using Gaussian elimination. A high level assumption is however needed to ensure that the Gaussian elimination performs adequately.

Cragg and Donald (1997) propose a rank statistic based on a minimum $\chi^{2}$ criterion. They assume that the rank of the covariance matrix of the unrestricted matrix is maximal and minimize the objective function numerically. Numerical optimization is often very difficult when the tested rank is less than the rank of the true value of the matrix. This results from the bad identification of the elements of the estimated matrix. The minimum of the criterium function is in this case not well-defined and the numerical optimization procedure may converge to an erroneous value.

Recently, Robin and Smith (2000) construct a rank statistic on the basis of a (root- $N$ ) consistent estimator for the unrestricted matrix. The covariance matrix of this estimator does not need to have the Kronecker structure and its rank does not have to be maximal. The limiting distribution of the rank statistic is, however, a weighted average of $\chi^{2}$ distributions. The asymptotic critical values of the rank statistic are therefore not tabulated. The limiting distribution shows that this rank statistic is not always appropriately normalized so the range of values of the weights affects the power of the statistic.

To overcome the deficiencies of the rank statistics, we propose a novel rank statistic. The rank statistic involves a (root- $N$ ) consistent estimator of the unrestricted matrix, which does not need to have a Kronecker covariance matrix. We decompose the unrestricted matrix estimator using a singular value decomposition. If the limiting distribution of the unrestricted matrix estimator is normal, an orthogonal transformation of the smallest singular values has a normal limiting distribution. Our rank statistic is equal to the quadratic form of this orthogonal transformation with respect to the inverse of its covariance matrix, and hence it has a standard $\chi^{2}$ limiting distribution. The rank statistic simplifies to the canonical correlation rank statistic of Anderson (1951) in case of a Kronecker covariance matrix. The rank statistic can also be applied in case of non-stationary variables, for example for cointegration testing. The asymptotic distribution is in that case a functional of Brownian motions and equal to the asymptotic distribution of the Johansen (1991) trace statistic.

The outline of the paper is as follows. In Section 2 we discuss the singular value decompo- 
sition of a matrix. We show how this decomposition can be used to obtain a parameter that reflects rank reduction. In Section 3 we construct a rank statistic based on this parameter. We derive its limiting distribution and show that the statistic is a generalization of the canonical correlation rank statistic. We limit ourselves in this section to stationary variables and compare the rank statistic with existing rank statistics. The non-stationary cointegration case is discussed in Section 4. In this case, the limit distribution of our rank statistic is identical to that of the Johansen trace statistic. In Section 5, we apply the rank statistic to test the rank of a matrix that governs the identification of the parameters in the stochastic discount factor model of Jagannathan and Wang (1996). Furthermore, we use a factor model which stems from this application to illustrate the validity of the limiting distribution and to conduct a power comparison of different rank statistics. We end with some concluding remarks and topics for further research in Section 6.

Throughout the paper we use the notation: $a=\operatorname{vec}(A)$ for the column vectorization of the $k \times m$ matrix $A$ such that for $A=\left(A_{1} \cdots A_{m}\right), \operatorname{vec}(A)=\left(A_{1}^{\prime} \cdots A_{m}^{\prime}\right)^{\prime}, I_{m}$ is the $m \times m$ identity matrix and $\operatorname{tr}(A)$ stands for the trace of a square matrix $A$. Furthermore, " $\vec{p}$ " stands for convergence in probability and " $\vec{d}$ " for convergence in distribution.

\section{Singular Value Decomposition and Rank Reduction}

To construct our rank test, we use that a $k \times m$ real matrix $C$ can be decomposed as

$$
C=A_{q} B_{q}+A_{q, \perp} \Lambda_{q} B_{q, \perp}
$$

with $A_{q}$ a $k \times q$ matrix, $B_{q}$ a $q \times m$ matrix, $\Lambda_{q}$ a $(k-q) \times(m-q)$ matrix, $A_{q, \perp}$ a $k \times(k-q)$ matrix, $B_{q, \perp}$ a $(m-q) \times m$ matrix and where $A_{q}^{\prime} A_{q, \perp} \equiv 0, B_{q, \perp} B_{q}^{\prime} \equiv 0, A_{q, \perp}^{\prime} A_{q, \perp} \equiv I_{k-q}$ and $B_{q, \perp} B_{q, \perp}^{\prime} \equiv I_{m-q}$ with $q<\min (k, m)$. If $\Lambda_{q}=0$, the rank of the matrix $C$ is determined by the rank of $A_{q} B_{q}$. If both $A_{q}$ and $B_{q}$ have full rank, the rank of $C$ is then equal to $q$. Our test for rank reduction will be based on a test for $\Lambda_{q}=0$. To obtain a value of $\Lambda_{q}$ which reflects the distance to rank reduction, we define $\Lambda_{q}$ as a transformation of the smallest singular values of the matrix $C$ as these values determine the rank of the matrix $C$ in a unambiguous way. In fact, to identify the matrices in decomposition (1) we link the decomposition to a singular value decomposition of $C$.

The singular value decomposition (SVD) of a real $k \times m$ matrix $C$ is given by

$$
C=U S V^{\prime}
$$

where $U$ is a $k \times k$ orthonormal matrix $\left(U^{\prime} U=I_{k}\right), V$ is a $m \times m$ orthonormal matrix $\left(V^{\prime} V=I_{m}\right)$, and $S$ is a $k \times m$ matrix that contains the singular values of $C$ on its main diagonal and is equal to zero elsewhere. If $k=m, S$ is a $k \times k$ diagonal matrix with the singular values in decreasing order on its main diagonal. If $k<m, S$ consists of a $k \times k$ diagonal matrix with the $k$ singular values on its main diagonal (in decreasing order) extended on the right hand side with a $k \times(m-k)$ matrix of zeros. If $k>m, S$ consists of a $m \times m$ diagonal matrix with the $m$ singular values on its main diagonal (in decreasing order) on top of a $(k-m) \times m$ matrix of zeros, see for example Golub and van Loan (1989) for details.

The expressions for $A_{q}, B_{q}$, and $\Lambda_{q}$ in terms of the smallest singular values of $C$ follow from the relation

$$
\left(\begin{array}{cc}
U_{11} & U_{12} \\
U_{21} & U_{22}
\end{array}\right)\left(\begin{array}{cc}
S_{1} & 0 \\
0 & S_{2}
\end{array}\right)\left(\begin{array}{cc}
V_{11}^{\prime} & V_{21}^{\prime} \\
V_{12}^{\prime} & V_{22}^{\prime}
\end{array}\right)=A_{q} B_{q}+A_{q, \perp} \Lambda_{q} B_{q, \perp}
$$


where $U_{11}, S_{1}$ and $V_{11}$ are $q \times q$ matrices, $U_{12}$ and $U_{21}^{\prime}$ are $q \times(k-q)$ matrices, $V_{12}$ and $V_{21}^{\prime}$ are $q \times(m-q)$ matrices, $U_{22}$ is a $(k-q) \times(k-q)$ matrix, $V_{22}$ a $(m-q) \times(m-q)$ matrix, and $S_{2}$ a $(k-q) \times(m-q)$ matrix. Equation (3) implies that

$$
A_{q} B_{q}=\left(\begin{array}{c}
U_{11} \\
U_{21}
\end{array}\right) S_{1}\left(\begin{array}{ll}
V_{11}^{\prime} & V_{21}^{\prime}
\end{array}\right) \text { and } A_{q, \perp} \Lambda_{q} B_{q, \perp}=\left(\begin{array}{c}
U_{12} \\
U_{22}
\end{array}\right) S_{2}\left(\begin{array}{cc}
V_{12}^{\prime} & V_{22}^{\prime}
\end{array}\right) .
$$

The exact relation between $U, S$ and $V$ depends on the specification of $A_{q}$ and $B_{q}$. If we do not normalize $A_{q} B_{q}$, the number of free elements in $A_{q}$ and $B_{q}(=k q+q m)$ is larger than the number of free elements of a $k \times m$ matrix with rank $q\left(=k m-q^{2}\right)$. Hence, if we want to solve for $A_{q}$ and $B_{q}$ we need to impose a normalization for $A_{q} B_{q}$. Several normalizations are possible and the normalization usually depends on the model at hand. As illustration we show the normalization involved in three econometric models that imply rank reduction, that is a. the linear instrumental variables regression model, b. the error correction cointegration model and c. a factor model.

a. The linear instrumental variables regression model for the $q$-dimensional endogenous variable $y_{1, i}$ and $(k-q)$-dimensional endogenous variable $y_{2, i}$ with the $m$-dimensional vector of instruments $z_{i}$

$$
\begin{aligned}
& y_{1, i}=B_{q} z_{i}+\varepsilon_{1, i} \\
& y_{2, i}=A_{q, 2} y_{1, i}+\varepsilon_{2, i}, \text { for } i=1, \ldots, N
\end{aligned}
$$

can be written as

$$
\left(\begin{array}{l}
y_{1, i} \\
y_{2, i}
\end{array}\right)=C z_{i}+\left(\begin{array}{l}
u_{1, i} \\
u_{2, i}
\end{array}\right)
$$

where $u_{1, i}=\varepsilon_{1, i}, u_{2, i}=\varepsilon_{2, i}+A_{q, 2} \varepsilon_{1, i}$, and $C=A_{q} B_{q}$ with normalization $A_{q}=\left[I_{q} \vdots A_{q, 2}^{\prime}\right]^{\prime}$.

b. In a vector autoregression of order 1 for the $k$-dimensional time series $\left\{y_{i}\right\}_{i=0}^{N}$ in error correction form

$$
\Delta y_{i}=C y_{i-1}+\varepsilon_{i},
$$

rank reduction of the $k \times k$ matrix $C$ implies cointegration, see for example Engle and Granger (1987) and Johansen (1991). If the cointegration rank is $q$, we can write (7) as

$$
\Delta y_{i}=A_{q} B_{q} y_{i-1}+\varepsilon_{i} .
$$

For economic interpretation of the cointegration relation $B_{q} y_{i}$, it is convenient to normalize $B_{q}$ as $\left[I_{q} \vdots B_{q, 2}\right]$, where $B_{q, 2}$ is a $q \times(k-q)$ matrix.

c. In a factor model, where one relates a $k$-dimensional vector of variables $r_{i}$ to a $m$ dimensional vector of explanatory variables $f_{i}$,

$$
r_{i}=C f_{i}+\varepsilon_{i}, \text { for } i=1, \ldots, N
$$

one typically imposes rank reduction on $C$ to reduce the number of parameters. If the rank of $C$ is $q$, we can write $C=A_{q} B_{q}$. Since there is often no clear interpretation of $B_{q} f_{i}$, one may impose that $B_{q}\left(\frac{1}{N} \sum_{i=1}^{N} f_{i} f_{i}^{\prime}\right) B_{q}^{\prime}=I_{q}$ as a normalization. 
Given an appropriate normalization, we can solve for $A_{q}$ and $B_{q}$ in (4). For example, if we impose that $B_{q}=\left[I_{q} \vdots B_{q, 2}\right]$ with $B_{q, 2}$ a $q \times(m-q)$ matrix, as in the cointegration model, the first equation in (4) implies that

$$
A_{q}=\left(\begin{array}{c}
U_{11} \\
U_{21}
\end{array}\right) S_{1} V_{11}^{\prime} \text { and } B_{q, 2}=\left(V_{11}^{\prime}\right)^{-1} V_{21}
$$

Although $\Lambda_{q}$ is initially only identified up to an orthonormal transformation, since $A_{q, \perp}^{*} \Lambda_{q}^{*} B_{q, \perp}^{*}=$ $A_{q, \perp} \Lambda_{q} B_{q, \perp}$ with $A_{q, \perp}^{*}=A_{q, \perp} R_{q}, \Lambda_{q}^{*}=R_{q}^{\prime} \Lambda_{q} Q_{q}^{\prime}, B_{q, \perp}^{*}=Q_{q} B_{q, \perp}$ and $R_{q}$ and $Q_{q}$ are $(k-q) \times$ $(k-q)$ and $(m-q) \times(m-q)$ orthonormal matrices, a unique specification of $\Lambda_{q}$ results when we use an appropriate normalization of $A_{q}$ and $B_{q}$. This normalization allows us to express $A_{q, \perp}$ and $B_{q, \perp}$ as functions of the unrestricted elements of $A_{q}$ and $B_{q}$ and hence in terms of $U_{11}, U_{21}, S_{1}, V_{11}$, and $V_{21}$. We show below that this leads to the expression of $\Lambda_{q}{ }^{1}$

$$
\Lambda_{q}=\left(U_{22} U_{22}^{\prime}\right)^{-\frac{1}{2}} U_{22} S_{2} V_{22}^{\prime}\left(V_{22} V_{22}^{\prime}\right)^{-\frac{1}{2}}
$$

which implies, given the second equation in (4), that

$$
A_{q, \perp}=\left(\begin{array}{c}
U_{12} \\
U_{22}
\end{array}\right) U_{22}^{-1}\left(U_{22} U_{22}^{\prime}\right)^{\frac{1}{2}} \text { and } B_{q, \perp}=\left(V_{22} V_{22}^{\prime}\right)^{\frac{1}{2}}\left(V_{22}^{\prime}\right)^{-1}\left[V_{12}^{\prime} \vdots V_{22}^{\prime}\right] \text {. }
$$

For (11) and (12), we can express $A_{q, \perp}$ in terms of $A_{q}=\left[A_{q, 1}^{\prime} \vdots A_{q, 2}^{\prime}\right]^{\prime}$ with $A_{q, 1}$ a $q \times q$ matrix and $A_{q, 2}$ a $(k-q) \times q$ matrix as

$$
\begin{aligned}
A_{q, \perp} & =\left(\begin{array}{c}
U_{12} U_{22}^{-1} \\
I_{k-q}
\end{array}\right)\left(\left(U_{22}^{\prime}\right)^{-1} U_{22}^{-1}\right)^{-\frac{1}{2}} \\
& =\left(\begin{array}{c}
U_{12} U_{22}^{-1} \\
I_{k-q}
\end{array}\right)\left(\left(U_{22}^{\prime}\right)^{-1}\left(U_{22}^{\prime} U_{22}+U_{12}^{\prime} U_{12}\right) U_{22}^{-1}\right)^{-\frac{1}{2}} \\
& =\left(\begin{array}{c}
U_{12} U_{22}^{-1} \\
I_{k-q}
\end{array}\right)\left(I_{k-q}+\left(U_{22}^{\prime}\right)^{-1} U_{12}^{\prime} U_{12} U_{22}^{-1}\right)^{-\frac{1}{2}} \\
& =\left(\begin{array}{c}
-\left(U_{11}^{\prime}\right)^{-1} U_{21}^{\prime} \\
I_{k-q}
\end{array}\right)\left(I_{k-q}+U_{21} U_{11}^{-1}\left(U_{11}^{\prime}\right)^{-1} U_{21}^{\prime}\right)^{-\frac{1}{2}} \\
& =\left(\begin{array}{c}
-\left(U_{11}^{\prime}\right)^{-1} U_{21}^{\prime} \\
I_{k-q}
\end{array}\right)\left(I_{k-q}+U_{21} S_{1} V_{11}^{\prime}\left(V_{11}^{\prime}\right)^{-1} S_{1}^{-1} U_{11}^{-1}\left(U_{11}^{\prime}\right)^{-1}\left(S_{1}^{\prime}\right)^{-1} V_{11}^{-1} V_{11} S_{1}^{\prime} U_{21}^{\prime}\right)^{-\frac{1}{2}} \\
& =\left(\begin{array}{c}
-\left(A_{q, 1}^{\prime}\right)^{-1} A_{q, 2} \\
I_{k-q}
\end{array}\right)\left(I_{k-q}+A_{q, 2} A_{q, 1}^{-1}\left(A_{q, 1}^{\prime}\right)^{-1} A_{q, 2}^{\prime}\right)^{-\frac{1}{2}}
\end{aligned}
$$

where we use the orthonormality properties of $U$ and $V$. Likewise, we can write $B_{q, \perp}$ in terms of $B_{q, 2}$

$$
\begin{aligned}
B_{q, \perp} & =\left(\left(V_{22}^{\prime}\right)^{-1} V_{22}^{-1}\right)^{-\frac{1}{2}}\left[\left(V_{22}^{\prime}\right)^{-1} V_{12}^{\prime} \vdots I_{m-q}\right] \\
& =\left(\left(V_{22}^{\prime}\right)^{-1}\left(V_{22}^{\prime} V_{22}+V_{12}^{\prime} V_{12}\right) V_{22}^{-1}\right)^{-\frac{1}{2}}\left[\left(V_{22}^{\prime}\right)^{-1} V_{12}^{\prime} \vdots I_{m-q}\right] \\
& =\left(I_{m-q}+\left(V_{22}^{\prime}\right)^{-1} V_{12}^{\prime} V_{12} V_{22}^{-1}\right)^{-\frac{1}{2}}\left[\left(V_{22}^{\prime}\right)^{-1} V_{12}^{\prime} \vdots I_{m-q}\right] \\
& =\left(I_{m-q}+V_{21}^{\prime} V_{11}^{-1}\left(V_{11}^{\prime}\right)^{-1} V_{21}\right)^{-\frac{1}{2}}\left[-V_{21}^{\prime} V_{11}^{-1} \vdots I_{m-q}\right] \\
& =\left(I_{m-q}+B_{q, 2}^{\prime} B_{q, 2}\right)^{-\frac{1}{2}}\left[-B_{q, 2}^{\prime} \vdots I_{m-q}\right]
\end{aligned}
$$

\footnotetext{
${ }^{1}$ If $D$ is a positive definite real symmetric matrix, then $D^{\frac{1}{2}}=E L E^{\prime}$, where $L$ is a diagonal matrix containing the square roots of the eigenvalues of $D, E$ contains the orthonormal eigenvectors of $D$, and $D^{-\frac{1}{2}}=E L^{-1} E^{\prime}$, see for example Johansen (1995, p. 222).
} 
We note that every normalization of $A_{q}$ and $B_{q}$ leads to the same expressions of $\Lambda_{q}, A_{q, \perp}$ and $B_{q, \perp}$ in (11) and (12). The matrices $\left(U_{22} U_{22}^{\prime}\right)^{-\frac{1}{2}} U_{22}$ and $V_{22}^{\prime}\left(V_{22} V_{22}^{\prime}\right)^{-\frac{1}{2}}$ in the expression of $\Lambda_{q}$ (11) are both orthonormal matrices. Hence, the matrix $\Lambda_{q}$ is equal to the matrix $S_{2}$, that contains the smallest singular values, pre- and postmultiplied by orthonormal matrices or stated differently, $\Lambda_{q}$ is just a rotation of the smallest singular values around the origin. The singular values are non-negative and therefore all elements of $S_{2}$ are also non-negative. If we pre- and post-multiply $S_{2}$ by orthonormal matrices, the elements of the resulting matrix $\Lambda_{q}$ are no longer restrained to be non-negative. Hence, when $C$ is a random matrix, the elements of $\Lambda_{q}$ can be normally distributed while the elements of $S_{2}$ can not be normally distributed. This property allows us to base tests for rank reduction with $\chi^{2}$ limiting distributions on $\Lambda_{q}$ as we show in the next section.

\section{Rank Test: The Standard (Stationary) Case}

We are concerned with testing the rank of the $k \times m$ matrix of parameters $\Pi$. Under our null hypothesis, the rank of the matrix $\Pi$ is equal to $q$ with $q<\min (k, m)$, that is, $\mathrm{H}_{0}$ : $\operatorname{rank}(\Pi)=q$. To derive the limiting distributions of our test statistics and estimators, we make an assumption about the limiting behavior of an estimator of the unrestricted value of $\Pi$ denoted by $\hat{\Pi}$, where we use the notation $\hat{\pi}=\operatorname{vec}(\hat{\Pi})$ and $\pi=\operatorname{vec}(\Pi)$.

Assumption 1. The limiting behavior of the estimator of $\Pi$ is characterized by

$$
\begin{gathered}
\sqrt{N}(\hat{\pi}-\pi) \underset{d}{\rightarrow} \psi_{\pi}, \\
\psi_{\pi} \sim N(0, V),
\end{gathered}
$$

where $N$ is the sample size and $V$ is a $\mathrm{km} \times \mathrm{km}$ covariance matrix.

If we apply the SVD given in (2) directly on $\hat{\Pi}$ to test for the rank of $\Pi$, the resulting procedure can be sensitive to scaling of $\hat{\Pi}$, that is, pre- or post-multiplying $\hat{\Pi}$ by some matrix can affect the outcome of the rank test. We therefore allow $\hat{\Pi}$ to be normalized before we conduct a SVD of it. We can normalize $\hat{\Pi}$ by means of pre-multiplication by a $k \times k$ finite non-singular matrix $G$ and post-multiplication by a $m \times m$ finite non-singular matrix $F$. We are free to choose the specification of $F$ and $G$. The matrices $F$ and $G$ adapt the scaling of $\hat{\Pi}$

$$
\hat{\Theta}=G \hat{\Pi} F^{\prime}
$$

such that $\hat{\theta}=\operatorname{vec}(\hat{\Theta})=(F \otimes G) \hat{\pi}$. Using an appropriate specification of $F$ and $G$, the estimator $\hat{\Theta}$ becomes invariant to invertible transformations of the data that are identical over all observations. The limiting behavior of $\hat{\theta}$ is characterized by

$$
\begin{gathered}
\sqrt{N}(\hat{\theta}-\theta) \underset{d}{\rightarrow} \psi_{\theta}, \\
\psi_{\theta} \sim N(0, W),
\end{gathered}
$$

where $W=(F \otimes G) V(F \otimes G)^{\prime}$ and $\theta=(F \otimes G) \pi$.

To test $\mathrm{H}_{0}: \operatorname{rank}(\Pi)=q$, which is equivalent to $\mathrm{H}_{0}: \operatorname{rank}(\Theta)=q$, we use decomposition (1) for $\Theta$

$$
\Theta=A_{q} B_{q}+A_{q, \perp} \Lambda_{q} B_{q, \perp},
$$


where the components follow from the $\operatorname{SVD}(2)$ as described in Section 2. Under $\mathrm{H}_{0}$, the $(k-q) \times(m-q)$ matrix $\Lambda_{q}$ is identical to zero. The null hypothesis $\mathrm{H}_{0}: \operatorname{rank}(\Theta)=q$ is identical to $\mathrm{H}_{0}: \Lambda_{q}=0$. To obtain $\Lambda_{q}$ from (18), we need to pre- and post-multiply $\Theta$ by $A_{q, \perp}^{\prime}$ and $B_{q, \perp}^{\prime}$, respectively. To test $\mathrm{H}_{0}: \Lambda_{q}=0$, we therefore have to make an assumption concerning the covariance matrix $W$.

Assumption 2. The $(k-q)(m-q) \times(k-q)(m-q)$ covariance matrix

$$
\Omega_{q}=\left(B_{q, \perp} \otimes A_{q, \perp}^{\prime}\right) W\left(B_{q, \perp} \otimes A_{q, \perp}^{\prime}\right)^{\prime}
$$

is non-singular.

Decomposition (18) is also applied to the estimator $\hat{\Theta}$,

$$
\hat{\Theta}=\hat{A}_{q} \hat{B}_{q}+\hat{A}_{q, \perp} \hat{\Lambda}_{q} \hat{B}_{q, \perp} .
$$

The limiting behavior of the different elements of $\hat{\Theta}$ in $(20)$ is stated in Theorem 1.

Theorem 1 Under $H_{0}$ and Assumptions 1-2, the limiting behavior of the elements of $\hat{\Theta}$ in (20) is such that $\hat{A}_{q} \hat{B}_{q}$ is a root- $N$ consistent estimator of $A_{q} B_{q}$ and

$$
\begin{gathered}
\sqrt{N} \hat{\lambda}_{q} \underset{d}{\rightarrow} \psi_{\lambda}, \\
\psi_{\lambda} \sim N\left(0, \Omega_{q}\right),
\end{gathered}
$$

where $\hat{\lambda}_{q}=\operatorname{vec}\left(\hat{\Lambda}_{q}\right)$ and $\hat{\Lambda}_{q}=\hat{A}_{q, \perp}^{\prime} \hat{\Theta} \hat{B}_{q, \perp}^{\prime}$.

Proof. see the Appendix.

We use $\hat{\lambda}_{q}$ to define the statistic to test $\mathrm{H}_{0}: \operatorname{rank}(\Theta)=q$.

Definition 1: Under Assumptions 1-2, the statistic

$$
\operatorname{rk}(q)=\frac{1}{N} \hat{\lambda}_{q}^{\prime} \Omega_{q}^{-1} \hat{\lambda}_{q}
$$

converges under $H_{0}: \operatorname{rank}(\Theta)=q$ in distribution to a $\chi^{2}((k-q)(m-q))$ random variable.

The statistic (22), to which we refer as the rk-statistic, becomes an operational statistic for testing the rank of $\Theta$ when we have specified a consistent estimator for the covariance matrix $\Omega_{q}$, which corresponds with a consistent estimator for the covariance matrix $V$. The rk-statistic therefore allows for the use of both parametric as well as non-parametric covariance matrix estimators, like, for example, HACC matrix estimators, see Andrews (1991), Newey and West (1987), West (1997) and White (1980).

The rk-statistic is related to the rank tests discussed in the literature. To illustrate this, we separately discuss the relationship between the rk-statistic and the rank statistics proposed by Anderson (1951), Robin and Smith (2000), Cragg and Donald (1997) and Gill and Lewbel (1992)-Cragg and Donald (1996).

Anderson (1951) canonical correlation rank statistic When the covariance matrix $V$ has a Kronecker form, the rk-statistic is identical to the canonical correlation rank statistic of Anderson (1951) when $F$ and $G$ are appropriately specified. 
Proposition 1. When $V=\left(\left(F^{\prime} F\right)^{-1} \otimes\left(G^{\prime} G\right)^{-1}\right)$, the rank statistic rk(q) (22) is identical to the canonical correlation rank statistic of Anderson (1951). When $k>m$, it is equal to the sum of the $m-q$ smallest eigenvalues of $\hat{\Theta}^{\prime} \hat{\Theta}$ divided by $N$, and when $m>k$, it is equal to the sum of the $k-q$ smallest eigenvalues of $\hat{\Theta} \hat{\Theta}^{\prime}$ divided by $N$. The smallest eigenvalues of $\hat{\Theta}^{\prime} \hat{\Theta}$ and $\hat{\Theta} \hat{\Theta}^{\prime}$ represent the smallest canonical correlations.

\section{Proof. see the Appendix.}

Proposition 1 shows that the rk-statistic is a generalization of the canonical correlation rank statistic of Anderson (1951). It generalizes the canonical correlation rank statistic to a more general specification of the covariance matrix $V$ with the same limiting distribution. A non-Kronecker structure of the covariance matrix $V$ occurs when we use an estimator $\hat{\Pi}$ that does not give the same weight to every observation or when the disturbances of the underlying model are heteroscedastic or correlated.

Robin and Smith (2000) propose a rank test which results from the limiting distribution of the roots of the polynomial

$$
\left|\hat{\Pi}^{\prime} G^{\prime} G \hat{\Pi}-\mu\left(F^{\prime} F\right)^{-1}\right|=0, \quad k \geq m
$$

or

$$
\left|\hat{\Pi} F^{\prime} F \hat{\Pi}^{\prime}-\mu\left(G^{\prime} G\right)^{-1}\right|=0, \quad m<k,
$$

which are identical to the eigenvalues of $\hat{\Theta}^{\prime} \hat{\Theta}$ and $\hat{\Theta} \hat{\Theta}^{\prime}$. Robin and Smith (2000) show that, under $\mathrm{H}_{0}: \operatorname{rank}(\Pi)=q$ and Assumptions 1 and 2, the limiting distribution of the statistic

$$
\operatorname{CRT}(q)=N \sum_{i=q+1}^{\min (k, m)} h\left(\hat{\mu}_{i}\right)
$$

where $h(z)$ is a continuous differentiable non-negative scalar function, $h(0)=0,\left.\frac{d h(z)}{d z}\right|_{z=0}=1$, and $\hat{\mu}_{1}>\hat{\mu}_{2}>\ldots>\hat{\mu}_{\min (k, m)}$ are the roots of the polynomial (23) or (24), is characterized by

$$
\sum_{i=1}^{(k-q)(m-q)} \tau_{i} \psi_{i}
$$

where $\psi_{i}, i=1, \ldots,(k-q)(m-q)$, are independent $\chi^{2}(1)$ random variables and $\tau_{i}, i=$ $1, \ldots,(k-q)(m-q)$, are the $(k-q)(m-q)$ eigenvalues of $\Omega_{q} \cdot{ }^{2}$ An example of a function $h$ is $h(z)=z$, for other examples we refer to Robin and Smith (2000).

The limiting distributions of the CRT and rk-statistics apply under the same conditions which indicates that these statistics are closely related. The relation between the two tests follows from the fact that the roots of polynomials (23) and (24) are equal to the squared singular values of $\hat{\Theta}$, since they are equal to the eigenvalues of $\hat{\Theta}^{\prime} \hat{\Theta}$ and $\hat{\Theta} \hat{\Theta}^{\prime}$. The smallest $\min (k-q, m-q)$ singular values are contained in $S_{2}$. Hence, Robin and Smith (2000) construct the limiting distribution of $\operatorname{tr}\left(S_{2}^{\prime} S_{2}\right)\left(=\operatorname{tr}\left(S_{2} S_{2}^{\prime}\right)\right)$. To construct our rk-statistic, we first conduct

\footnotetext{
${ }^{2}$ Robin and Smith (2000) express the eigenvectors of the roots of the polynomials (23) and (24) normalized with respect to either $\left(F^{\prime} F\right)^{-1}$ and $\left(G^{\prime} G\right)^{-1}$ so $D_{k-q}^{\prime}\left(G^{\prime} G\right)^{-1} D_{k-q}=I_{k-q}$ and $E_{m-q}^{\prime}\left(F^{\prime} F\right)^{-1} E_{m-q}=I_{m-q}$ where the $k \times(k-q)$ and $m \times(m-q)$ matrices $D_{k-q}$ and $E_{m-q}$ contain the eigenvectors that belong to the smallest roots of the polynomials $(24)$ and (23) respectively. The matrix $\left(E_{m-q} \otimes D_{k-q}\right)^{\prime} V\left(E_{m-q} \otimes D_{k-q}\right)$ that results from Theorem 3.2 of Robin and Smith (2000) is therefore identical to $\Omega_{q}$ in Assumption 2 since $E_{m-q}=\left(B_{q, \perp} F\right)^{\prime}$ and $D_{k-q}=G A_{q, \perp}$.
} 
an orthogonal transformation from $S_{2}$ to $\Lambda_{q}$. Because $\Lambda_{q}$ has a normal limiting distribution we can obtain its asymptotic covariance matrix. Since $S_{2}$ has only non-negative elements, it does not have a normal limiting distribution and we can not construct the asymptotic covariance matrix of $S_{2}$. We use the asymptotic covariance matrix of $\Lambda_{q}$ to normalize $\Lambda_{q}$ which leads to a statistic with a standard $\chi^{2}$ limiting distribution. Since $S_{2}$ is not appropriately normalized in $\operatorname{tr}\left(S_{2}^{\prime} S_{2}\right)$ and $\operatorname{tr}\left(S_{2} S_{2}^{\prime}\right)$, the limiting distribution of the CRT-statistic is not a standard $\chi^{2}$ one. It consists of a weighted sum of $\chi^{2}(1)$ random variables. The weights result from the asymptotic covariance matrix of $\Lambda_{q} \operatorname{since} \operatorname{tr}\left(S_{2}^{\prime} S_{2}\right)=\operatorname{tr}\left(\Lambda_{q}^{\prime} \Lambda_{q}\right)$. The critical values of the limiting distribution of the CRT-statistic are therefore not readily available, and given the value of the weights we have to simulate from the limiting distribution to obtain them. Because $S_{2}$ is not appropriately normalized in the CRT-statistic, the $\tau_{i}, i=1, \ldots,(k-q)(m-q)$ are of importance for the power of the rank test. When there is a large difference between the different $\tau_{i}, i=1, \ldots,(k-q)(m-q)$, the CRT-statistic essentially only focusses on the large $\tau_{i}$ since the small $\tau_{i}$ will not contribute much to the critical value. The rk-statistic normalizes $\Lambda_{q}$ using its asymptotic covariance matrix and hence it focusses on all elements of $S_{2}$ in a more evenly distributed manner.

When $V=\left(\left(F^{\prime} F\right)^{-1} \otimes\left(G^{\prime} G\right)^{-1}\right)$, the values of $\tau_{i}$ are equal to one and the limiting distribution of the CRT-statistic is a $\chi^{2}((k-q)(m-q))$ distribution. In this case, the CRT-statistic is also identical to the canonical correlation rank statistic of Anderson (1951).

Cragg and Donald (1997) construct a rank statistic by means of the minimal value of a criterium function,

$$
\mathrm{CD}(q)=\min _{\Pi_{0} \in \Gamma(q)} N\left(\hat{\pi}-\pi_{0}\right)^{\prime} V^{-1}\left(\hat{\pi}-\pi_{0}\right),
$$

where $\pi_{0}=\operatorname{vec}\left(\Pi_{0}\right)$ and $\Gamma(q)$ is the space of $k \times m$ matrices with rank less than or equal to $q$. Cragg and Donald (1997) show that the CD-statistic $(27)$ has a $\chi^{2}((k-q)(m-q))$ limiting distribution. Unless $V$ has a Kronecker structure, in which case the CD-statistic corresponds with the canonical correlation rank statistic, $\Pi_{0}$ can not be obtained analytically and a numerical optimization procedure has to be used. To test for the rank of $\Pi$, we compute $\operatorname{CD}(q)$ sequentially for values of $q$ equal to $0,1, \ldots, \min (k, m)$. Eventually $\operatorname{CD}(q)$ is therefore computed at some value of $q$ that exceeds the rank of $\Pi$. For these values of $q$, the value of $\Pi_{0}$ that minimizes (27) is often not well-defined since some elements of $\Pi_{0}$ hardly influence the objective function. This holds especially when $k$ and/or $m$ are rather large. Hence, the numerical optimization procedure may return a erroneous value of $\Pi_{0}$ that results from an area where the objective function is rather flat. This is a deficiency of the numerical optimization that is involved in the CD-statistic. Another difference with the rk-statistic concerns the covariance matrix $V$ which has to be non-singular while the rk-statistic only assumes a nonsingular covariance matrix $\Omega_{q}$. The latter assumption is a less restrictive.

Gill and Lewbel (1992)-Cragg and Donald (1996) Alongside the singular value decomposition, another well-known matrix decomposition is the LDU-decomposition, see for example Golub and van Loan (1989). Gill and Lewbel (1992) and Cragg and Donald (1996) use the LDU-decomposition to construct statistics to test $\mathrm{H}_{0}: \operatorname{rank}(\Pi)=q$. To construct these LDU-statistics, permutations are conducted on $\Pi$ which transform it into $\Theta$,

$$
\Theta=G \Pi F^{\prime}
$$

where $G$ and $F$ are $k \times k$ and $m \times m$ permutation matrices that, in case we want to test $\mathrm{H}_{0}$ : $\operatorname{rank}(\Theta)=q$, are such that the first $q$ columns and rows of $\Theta$ perform a Gaussian elimi- 
nation of $\Pi$. For the LDU-decomposition, $\Theta$ is specified as

$$
\Theta=L D U
$$

with $L: k \times k, D: k \times m$ and $U: m \times m$ matrices that are specified by

$$
L=\left(\begin{array}{cc}
L_{11} & 0 \\
L_{21} & I_{k-q}
\end{array}\right), \quad D=\left(\begin{array}{cc}
D_{11} & 0 \\
0 & D_{22}
\end{array}\right), \quad U=\left(\begin{array}{cc}
U_{11} & U_{12} \\
0 & I_{m-q}
\end{array}\right)
$$

where $L_{11}, D_{11}, U_{11}: q \times q ; L_{21}:(k-q) \times q ; D_{22}:(k-q) \times(m-q) ; U_{12}: q \times(m-q)$; and $L_{11}$ and $U_{11}^{\prime}$ are lower triangular matrices with ones on the diagonal and $D_{11}$ is a diagonal matrix. ${ }^{3}$ The Gaussian elimination accomplishes that the largest elements of $\Theta$ are grouped in $D_{11}, L_{11}, L_{21}, U_{11}$ and $U_{12}$ and the smallest in $D_{22}$. Under $H_{0}: \operatorname{rank}(\Theta)=q, D_{22}$ is therefore equal to zero.

To construct the LDU-statistic to test $\mathrm{H}_{0}: \operatorname{rank}(\Theta)=q$, we conduct the same LDUdecomposition with Gaussian elimination (28)-(30) on $\hat{\Pi}$ as on $\Pi$. Cragg and Donald (1996) show that under $\mathrm{H}_{0}: \operatorname{rank}(\Theta)=q$, Assumptions 1, 2, and an assumption on the Gaussian elimination that $\operatorname{vec}\left(\hat{D}_{22}\right)$ has a normal limiting distribution with mean zero. The LDUstatistic is equal to a quadratic form of $\operatorname{vec}\left(\hat{D}_{22}\right)$ with the inverse of its asymptotic covariance matrix and converges under $\mathrm{H}_{0}$ and the before-mentioned assumptions to a $\chi^{2}((k-q)(m-q))$ distributed random variable.

The LDU-decomposition is not unique and different orderings of the data result in different LDU-decompositions. Before the LDU-decomposition, Cragg and Donald (1996) therefore permute $\hat{\Pi} q$ times using Gaussian elimination. The Gaussian elimination re-orders the columns and rows of $\hat{\Pi}$ such that the first $q$ rows and columns of $\hat{\Theta}$ contain the largest elements of $\hat{\Pi}$. The Gaussian elimination accomplishes that the elements of $\hat{D}_{11}$ are significantly different from zero under $\mathrm{H}_{0}$. In that case, $\hat{D}_{22}$ can be used to test $\mathrm{H}_{0}: \operatorname{rank}(\Theta)=q$. Because of the triangular structure of $\hat{L}$ and $\hat{U}$ with ones on the diagonal, both $\hat{D}_{11}$ and $\hat{D}_{22}$ reflect whether $\hat{\Theta}$ is close to a lower rank value. The Gaussian elimination is needed to ensure that only $\hat{D}_{22}$ reflects whether $\operatorname{rank}(\hat{\Theta})$ is close to $q$. This explains why Cragg and Donald (1996) need an additional assumption, alongside Assumptions 1 and 2, to ensure that the Gaussian elimination performs adequately. Since this assumption is not made for the rk-statistic, the LDU-statistic applies in a more restricted setting than the rk-statistic although their limiting distributions are identical.

\section{Rank Test: The Non-Stationary Cointegration Case}

In vector autoregressive (VAR) models, cointegration implies a reduced rank value of the long-run multiplier, see for example Engle and Granger (1987) and Johansen $(1991,1995)$. A convenient specification of a $\operatorname{VAR}(1)$ model to reflect cointegration is the so-called error correction specification

$$
\Delta y_{i}=\Pi y_{i-1}+\varepsilon_{i}, \text { for } i=1, \ldots, N,
$$

where $y_{i}$ is a $k \times 1$ vector that contains time-series observations of the variable $y$ at time $i$, $\Delta y_{i}=y_{i}-y_{i-1}, \varepsilon_{i}$ is a $k \times 1$ vector of disturbances which we assume to be uncorrelated over

\footnotetext{
${ }^{3}$ In the LDU-decomposition that is used by Gill and Lewbel (1992), the matrices $L_{22}$ and $U_{22}$, which are identity matrices in (30), are also lower triangular with ones on the diagonal and $D_{22}$ is a diagonal matrix. Cragg and Donald (1996) show that the estimator of $D_{22}, \hat{D}_{22}$, in this case has not a normal limiting distribution under $\mathrm{H}_{0}: \operatorname{rank}(\Theta)=q$. We therefore use the LDU-decomposition that is proposed by Cragg and Donald (1996).
} 
time. Cointegration occurs when the $k \times k$ matrix $\Pi$ has a reduced rank value, which we can denote as $\Pi=A_{q} B_{q}$ with $A_{q}$ a $k \times q$ matrix and $B_{q}$ a $q \times k$ matrix. The canonical correlation rank test for the rank of the long-run multiplier is in this case identical to the Johansen trace statistic, see Johansen (1991). These statistics apply when the covariance matrix of the disturbances $\varepsilon_{i}$ is constant over time.

In Kleibergen and Van Dijk (1994), the LDU decomposition is used to decompose the long-run multiplier $\hat{\Pi}$ to construct an alternative cointegration test. This cointegration test has however the same variable ordering problem as the LDU-based test in the stationary case. Therefore we construct in this paper a test for rank reduction in case of non-stationary variables, where we follow the same approach as in the previous section. Because the dependent and explanatory variables are different realizations of the same (economic) variables over time, we do not need to normalize the estimator $\hat{\Pi}$ to obtain a rank statistic. As mentioned before, an appropriately normalized rank statistic corresponds with the canonical correlation rank test that is identical to the Johansen trace statistic. We use the SVD to decompose the unrestricted least squares estimator $\hat{\Pi}=\sum_{i=1}^{N} \Delta y_{i} y_{i-1}^{\prime}\left(\sum_{i=1}^{N} y_{i-1} y_{i-1}^{\prime}\right)^{-1}$ as

$$
\hat{\Pi}=\hat{A}_{q} \hat{B}_{q}+\hat{A}_{q, \perp} \hat{\Lambda}_{q} \hat{B}_{q, \perp}
$$

The rank statistic corresponds to a quadratic form in $\lambda_{q}=\operatorname{vec}\left(\Lambda_{q}\right)$. To derive the limiting distribution of the rank statistic, we use the Representation Theorem for cointegrated series, see for example Johansen $(1991,1995)$. Theorem 2 states the limiting behavior of the rank statistic under the assumptions that the disturbances $\varepsilon_{i}$ are a white noise process with constant covariance matrix $\Sigma$.

Theorem 2 When the disturbances $\varepsilon_{i}$ are white noise with constant covariance matrix $\Sigma$, $\Pi=A_{q} B_{q}$ and $A_{q, \perp}^{\prime} B_{q, \perp}^{\prime}$ is non-singular, the limiting behavior of the rank statistic

$$
r k(q)=\frac{1}{N} \hat{\lambda}_{q}^{\prime} \hat{\Omega}_{q}^{-1} \hat{\lambda}_{q}
$$

with $\hat{\lambda}_{q}=\operatorname{vec}\left(\hat{\Lambda}_{q}\right)$,

$$
\hat{\Omega}_{q}=\left(\hat{B}_{q, \perp} \otimes \hat{A}_{q, \perp}^{\prime}\right) \hat{W}\left(\hat{B}_{q, \perp} \otimes \hat{A}_{q, \perp}^{\prime}\right)^{\prime}
$$

and

$$
\hat{W}=\left(\left(\frac{1}{N} \sum_{i=1}^{N} y_{i-1} y_{i-1}^{\prime}\right)^{-1} \otimes \frac{1}{N} \sum_{i=1}^{N} \tilde{\varepsilon}_{i} \tilde{\varepsilon}_{i}^{\prime}\right)
$$

where $\tilde{\varepsilon}_{i}=\Delta y_{i}-\hat{\Pi} y_{i-1}$, is characterized by

$$
r k(q) \underset{d}{\rightarrow} \operatorname{tr}\left[\left(\int_{0}^{1} B_{k-q}(t) d B_{k-q}(t)^{\prime}\right)^{\prime}\left(\int_{0}^{1} B_{k-q}(t) B_{k-q}(t)^{\prime} d t\right)^{-1}\left(\int_{0}^{1} B_{k-q}(t) d B_{k-q}(t)^{\prime}\right)\right]
$$

where $B_{k-q}(t)$ is a $(k-q)$-dimensional Brownian motion defined on the unit interval with identity covariance matrix at time $t$.

\section{Proof. see the Appendix.}

The limiting distribution in Theorem 2 is identical to the limiting distribution of the Johansen trace statistic, see Johansen $(1991,1995)$. The rank statistic (33) results directly from a SVD of the least squares estimator. It can therefore be considered as a multivariate generalization of the Dickey-Fuller $t$-statistic for testing unit roots in univariate autoregressive 
models. The Johansen trace statistic is a likelihood ratio statistic while the Dickey-Fuller statistic and the rank statistic (33) are Wald statistics.

The derivation of the limiting distribution of the rank test in the Appendix shows that we can also obtain the rank test from

$$
\begin{aligned}
\operatorname{rk}(q)= & \frac{1}{N}\left[\left(\hat{B}_{q, \perp} \otimes \hat{A}_{q, \perp}^{\prime}\right) \operatorname{vec}\left(\sum_{i=1}^{N} \hat{\varepsilon}_{i} y_{i-1}^{\prime}\right)\right]^{\prime}\left[\left(\hat{B}_{q, \perp} \otimes \hat{A}_{q, \perp}^{\prime}\right) \hat{V}\left(\hat{B}_{q, \perp} \otimes \hat{A}_{q, \perp}^{\prime}\right)^{\prime}\right]^{-1} \\
& {\left[\left(\hat{B}_{q, \perp} \otimes \hat{A}_{q, \perp}^{\prime}\right) \operatorname{vec}\left(\sum_{i=1}^{N} \hat{\varepsilon}_{i} y_{i-1}^{\prime}\right)\right] }
\end{aligned}
$$

where $\hat{\varepsilon}_{i}=\Delta y_{i}-\hat{A}_{q} \hat{B}_{q} y_{i-1}$ and $\hat{V}=\left(\frac{1}{N} \sum_{i=1}^{N} y_{i-1} y_{i-1}^{\prime} \otimes \frac{1}{N} \sum_{i=1}^{N} \tilde{\varepsilon}_{i} \tilde{\varepsilon}_{i}^{\prime}\right)$. Based on this specification of the rank test, we can define a set of assumptions, which are more general than the white noise constant variance assumption, under which the test has the same limiting distribution as the Johansen trace statistic.

Assumption 3. The limiting behavior of $\operatorname{vec}\left(\sum_{i=1}^{N} \hat{\varepsilon}_{i} y_{i-1}^{\prime}\right)$ is such that

$$
\frac{1}{N}\left[\left(\hat{B}_{q, \perp} \otimes \hat{A}_{q, \perp}^{\prime}\right) \operatorname{vec}\left(\sum_{i=1}^{N} \hat{\varepsilon}_{i} y_{i-1}^{\prime}\right)\right] \underset{d}{\rightarrow} \Psi_{q}^{\frac{1}{2}}\left(\int_{0}^{1} B_{k-q}(t) d B_{k-q}(t)^{\prime}\right)^{\prime} \Sigma_{q}^{\frac{1}{2}}\left(A_{q, \perp}^{\prime} B_{q, \perp}^{\prime}\right)^{-1 \prime},
$$

while the limiting behavior of $\hat{\Omega}_{q}=\left(\hat{B}_{q, \perp} \otimes \hat{A}_{q, \perp}^{\prime}\right) \hat{V}\left(\hat{B}_{q, \perp} \otimes \hat{A}_{q, \perp}^{\prime}\right)^{\prime}$ accords with

$$
\frac{1}{N} \hat{\Omega}_{q} \underset{d}{\rightarrow}\left(\left(A_{q, \perp}^{\prime} B_{q, \perp}^{\prime}\right)^{-1} \Sigma_{q}^{\frac{1}{2}}\left(\int_{0}^{1} B_{k-q}(t) B_{k-q}(t)^{\prime} d t\right) \Sigma_{q}^{\frac{1}{2}}\left(A_{q, \perp}^{\prime} B_{q, \perp}^{\prime}\right)^{-1 \prime} \otimes \Psi_{q}\right),
$$

where $\Sigma_{q}$ and $\Psi_{q}$ are $(k-q) \times(k-q)$ dimensional non-singular matrices.

Under Assumption 3, the rank statistic (37) has the limiting distribution given in (36). Assumption 3 allows for a more general set of processes that generate the disturbances $\varepsilon_{i}$ than white noise with a fixed covariance matrix. For example, as shown in Phillips and Solo (1992), these limiting distributions apply for processes with time-varying conditional variances for which the unconditional variance is finite and fixed. The rank statistic (37) therefore allows for the use of HACC matrix estimators $\hat{V}$, like the ones proposed by White (1980) and Newey and West (1987). In Andrews (1991), it is show that these covariance matrix estimators are consistent in case of non-stationary variables.

The decomposition in (32) can also be applied to other estimators of the long-run multiplier, for instance to those that result when we incorporate higher order lags or use different weights for the observations to account for time varying conditional variances or non-normal distributed errors.

\section{$5 \quad$ Testing rank in stochastic discount factor models}

Jagannathan and Wang (1996) base the stochastic discount factor (SDF) in a SDF model on a conditional Capital Asset Pricing Model (CAPM). A SDF model for asset returns is represented by, see for example Campbell et. al. (1997),

$$
E\left[r_{j i+1} s_{i+1} \mid I_{i}\right]=1, \quad j=1, \ldots, k,
$$

where $r_{j i}$ is the return at time $i$ on asset $j, j=1, \ldots, k, I_{i}$ is the information set at time $i$ and $s_{i}$ is the SDF at time $i$. Based on the conditional CAPM, Jagannathan and Wang (1996) specify the SDF $s_{i+1}$ as a linear function of a set of additional variables:

$$
s_{i+1}=f_{i+1}^{\prime} \gamma,
$$


where $f_{i+1}$ is a $m \times 1$ vector of additional variables and $\gamma$ is a $m \times 1$ vector of risk premia. We substitute the specification of the SDF (41) into the SDF model (40) to obtain

$$
\left\{E\left[r_{i+1} f_{i+1}^{\prime} \mid I_{i}\right]\right\} \gamma=\iota_{k},
$$

where $r_{i}=\left(r_{1 i} \ldots r_{k i}\right)^{\prime}$ and $\iota_{k}$ is a $k \times 1$ vector of ones. The vector of risk premia $\gamma$ can be estimated using Hansen's (1982) Generalized Method of Moments (GMM), see for example Jagannathan et. al. (2002). The GMM estimator of $\gamma$ is consistent if the $k \times m$ matrix

$$
E\left[r_{i+1} f_{i+1}^{\prime} \mid I_{i}\right]
$$

has full rank, see Hansen (1992), Newey and McFadden (1994) and Wright (2003). Tests of the rank of (43) therefore indicate whether all elements of $\gamma$ are identified.

The asset returns that are used in SDF models are typically returns on portfolios. These portfolios are constructed using some kind of criterium, like, for example, the market capitalization or size of stocks. It is well known now that the returns on portfolios that consist of small firm stocks exhibit autocorrelation, see for example Campbell et. al. (1997). This implies that the canonical correlation rank statistic to test for the rank of (43) is essentially inappropriate since the (asymptotic) covariance matrix of an estimator of (43) does not possess a Kronecker structure. In the next sub-sections, we therefore use the previously discussed rank tests, which can be applied when the covariance matrix does not have a Kronecker structure, to test for the rank of (43) in SDF models.

\subsection{Application}

Jagannathan and Wang (1996) construct return series on hundred size and beta sorted portfolios to estimate the risk premia in the SDF model (40). The return series begin in July 1963 and end in December 1990 so $N=330$. The SDF (41) is a linear function of a constant, the return on a value-weighted portfolio, a corporate bond yield spread and per capita labor income growth. The size and beta sorted portfolios are obtained by ranking all non-financial stocks listed in the NYSE and AMEX and covered by CRSP with respect to size. For every decile of size sorted stocks, the stocks are once more sorted with respect to their beta, that is their correlation with the market return. All stocks in such a beta sorted decile constitute one portfolio. Since we have both ten size and beta sorted deciles, the total number of portfolios equals hundred. Inverting a $100 \times 100$ leads to a large numerical error. To decrease the number of portfolios, we therefore regroup the beta sorted portfolios by using the average returns over all beta sorted portfolios within each size decile. This reduced the number of portfolios to ten which are sorted with respect to size.

When the expectation of $r_{i+1} f_{i+1}^{\prime}$ does not depend on the information set $I_{i}$, the expectation in (43) is a unconditional expectation. Instead of testing the rank of (43), we can then also analyze the rank of the $k \times m$ matrix $\Pi$ in a linear factor model (9) for the returns on the ten size sorted portfolios,

$$
r_{i}=\Pi f_{i}+\varepsilon_{i}, \quad i=1, \ldots, N,
$$

where $k=10, f_{i}=\left(f_{1 i} \ldots f_{m i}\right)^{\prime}$ with $m=4, N=330$, and the $k \times 1$ vector $\varepsilon_{i}$ contains the disturbances. The returns contained in $r_{i}$ are such that $r_{1 i}$ is the return on the smallest size portfolio and $r_{k i}$ is the return on the largest size portfolio at time $i$. The factors in $f_{i}$ consist of a constant term, a return on a value-weighted portfolio, a corporate bond yield spread and a measure of per capita labor income growth. 
Returns on portfolios of small firm stocks are known to exhibit autocorrelation, see for example Campbell et. al. (1997). We test for first order autocorrelation in the disturbances using a Lagrange multiplier (LM) statistic. The LM statistic results from regressing the residuals on the lagged residuals. The LM statistic equals 141.4 which is well above the $5 \%$ significance level of the $\chi^{2}\left(10^{2}\right)$ distribution that is equal to 124.2 . The (asymptotic) significance of the LM statistic for first order autocorrelation indicates that we have to be careful with interpreting the canonical correlation rank statistic. Alongside the canonical correlation rank statistic, we therefore compute rank statistics that allow for non-Kronecker covariance matrices. Using a consistent covariance matrix estimator, the limit distributions of these rank statistics also apply when the disturbances exhibit autocorrelation. The rank statistics that we compute are the LDU rank statistic, the rk-statistic (22) and the CD-statistic (27). We do not consider the rank statistic of Robin and Smith (2000) because, as shown in Section 3, it consists of exactly the same elements as the rk-statistic and has a non-standard limiting distribution which makes a direct comparison more difficult.

To compute the rank statistics, we first transform or normalize the least squares estimator of $\Pi, \hat{\Pi}=\sum_{i=1}^{N} r_{i} f_{i}^{\prime}\left(\sum_{i=1}^{N} f_{i} f_{i}^{\prime}\right)^{-1}$, towards $\hat{\Theta}=G \hat{\Pi} F$ (16) by using a specification of $F$ and $G$ corresponding to

$$
\left(\left(\sum_{i=1}^{N} f_{i} f_{i}^{\prime}\right)^{-1} \otimes \sum_{i=1}^{N} r_{i} r_{i}^{\prime}\right)=\left(\left(F^{\prime} F\right)^{-1} \otimes\left(G^{\prime} G\right)^{-1}\right)
$$

such that the covariance matrix estimator for $\hat{\theta}$ becomes

$$
\hat{W}=(F \otimes G) \hat{V}(F \otimes G)^{\prime},
$$

with $\hat{V}$ the covariance matrix estimator for $\hat{\pi}$. When the covariance matrix estimator $\hat{V}$ accords with (45), the rk-statistic is identical to the canonical correlation rank statistic, as in Proposition 1. Hence, the specification of $F$ and $G$ is such that the rk-statistic uses the same elements as the canonical correlation rank statistic to discriminate between different rank values of $\Theta$ but with a consistent covariance matrix estimator. We therefore consider the rk-statistic with this specification of $F$ and $G$ as a size-corrected canonical correlation rank statistic. We use HACC matrix estimators for the covariance matrix estimator $\hat{V} .^{4}$

Table 1 lists the values of statistics for testing the rank of $\Pi$ using different HACC matrix estimators. The LDU rank statistic results from the same specification of $\hat{\Theta}$ as we used for the rk-statistic. The applied HACC matrix estimators are the Newey-West (1987) estimator with one lag, the White (1980) estimator and the West (1997) estimator with one lag. The latter estimator is intended for moving average processes.

The values of the rank statistics in Table 1 indicate that rank zero and rank one for $\Pi$ are rejected with $5 \%$ asymptotic significance. The value of the LDU-statistic to test for rank two is quite different from the rank value of the other statistics. This is due to the sensitivity of the LDU-statistic to the ordering of the variables. Depending on the ordering of the series in $f_{i}$, the values of the rank tests can differ substantially. This explains why Cragg and Donald (1996) permute $\hat{\Theta}$ using Gaussian elimination. All other statistics are insensitive to the ordering of the variables. The CD-statistic (27) is obtained using numerical optimization. The rank two and rank three cases of the CD-statistic differ considerably from the corresponding other statistics. The other rank statistics are not asymptotically significant

\footnotetext{
${ }^{4}$ The specification of $F$ and $G$ that transforms $\hat{V}$ towards $\hat{W}$ is also convenient to control the numerical error. The covariance matrix $\hat{V}$ is a $40 \times 40$ matrix which can be difficult to invert when it is not scaled appropriately. This is achieved by using the specification of $F$ and $G$ from (45).
} 


\begin{tabular}{|c|c|c|c|c|c|c|c|}
\hline \multirow[b]{2}{*}{ rank } & \multicolumn{6}{|c|}{ test statistic } & \multirow[b]{2}{*}{$5 \% \mathrm{CV}$} \\
\hline & rk-NW & rk-White & rk-West & LDU-West & CD-West & can. cor. & \\
\hline 0 & 102.8 & 116.5 & 108.9 & 108.9 & 108.9 & 366.7 & 55.7 \\
\hline 1 & 40.42 & 41.85 & 44.1 & 43.8 & 44.1 & 40.96 & 40.1 \\
\hline 2 & 17.66 & 18.48 & 18.8 & 22.3 & 64.3 & 18.7 & 26.3 \\
\hline 3 & 6.79 & 7.33 & 6.40 & 8.02 & 57.9 & 8.66 & 14.1 \\
\hline
\end{tabular}

Table 1: Rank statistics with different covariance matrix estimators (can. cor. stands for canonical correlation, NW stands for Newey-West and CV stands for critical value).

at these rank values. The numerical optimization procedure that we employed, which is a iterative procedure that estimates the parameters conditional on one another, does therefore not converge to a well-defined optimum because, as indicated by the other rank statistics, such an optimum is not clearly present. This shows that the need for numerical optimization to construct the CD-statistic is a drawback for practical purposes because these numerical procedures perform badly when the estimated model has a rank value that exceeds the rank of the model that generated the data.

\subsection{Size Comparison}

The canonical correlation rank statistic that tests for rank one is approximately equal to its asymptotic 5\% significance level. Because of the autocorrelated disturbances, the critical value is, however, inappropriate such that we can not assess its critical value. To assess the validity of the asymptotic critical values of the different rank statistics from Table 1, we bootstrapped the distributions of these statistics. We estimate the parameters of the factor model (44) with disturbances that are first order moving averages. The estimated model is used as the data generating process to obtain the distributions of the rank statistics. The true value of $\Pi$ in the data generating process is the canonical correlation estimate of $\Pi$ with rank one and we use the same values for $f_{i}, i=1, \ldots, N$ as in the original model. The disturbances in the model are simulated from a normal distribution with mean zero, where we incorporate their moving average property in the data generating process.

Figure 1 shows the empirical distribution functions of the rank statistics obtained from the outlined data generating process. Figure 1 shows that the distribution of the canonical correlation rank statistic lies below its asymptotic distribution which is a $\chi^{2}(27)$ distribution. The empirical distribution functions of the rank statistics that employ the White and West covariance matrix estimators, that is, the rk-West, rk-White, CD-West and LDU-West, lie more on less on top of their asymptotic distribution function. This indicates that these HACC estimators perform adequately in restoring the distribution of the canonical correlation rank statistic. The empirical distribution function of the rk-statistic with the Newey-West covariance matrix estimator lies above its asymptotic distribution. For this data generating process, the Newey-West covariance matrix estimator therefore performs less satisfactory than the West and White covariance matrix estimators.

Figure 1 supports the validity of the asymptotic critical values for the rank statistics with HACC matrix estimators reported in Table 1. We conclude from Table 1 that we can not reject rank values of $\Pi$ equal to two and three. Hence, we can not reject lower rank values of $\Pi$ and also not the non-identifiedness of (elements of) the vector of risk premia $\gamma$. GMM estimates of $\gamma$ can therefore be estimates of non-identified parameters which implies that their value can be more or less anything. This is reflected by the value and standard error (s.e.) of 


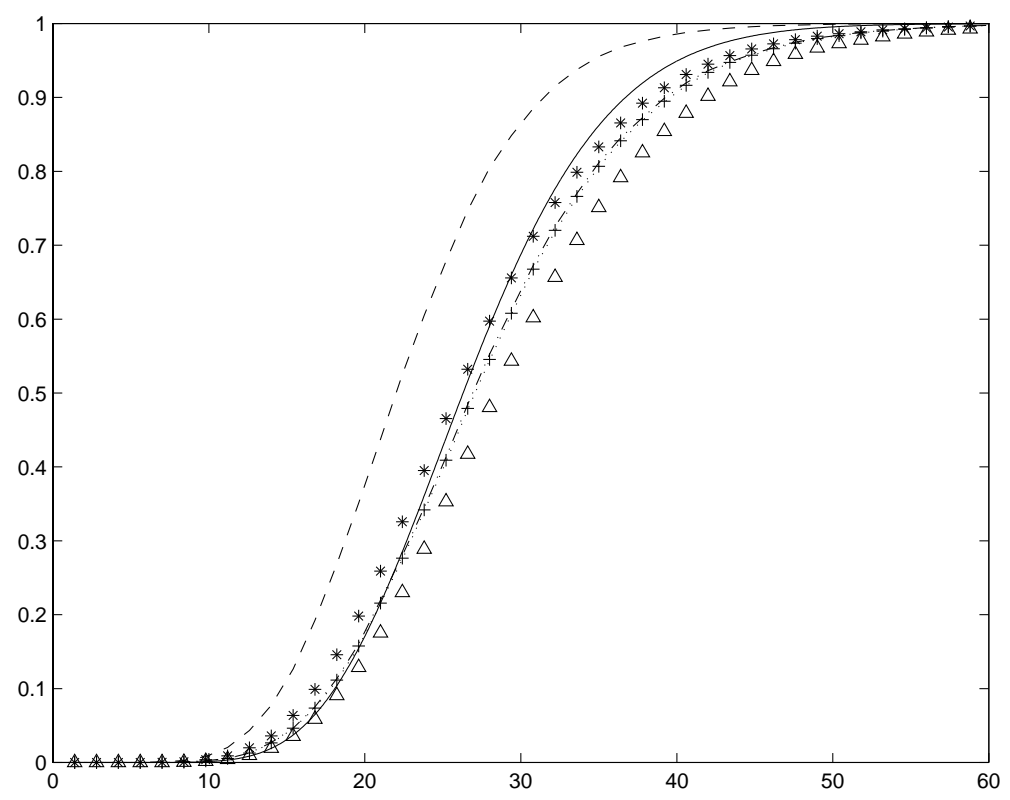

Figure 1: Empirical distribution functions of rank statistics that test for rank one while true rank is equal to one: asymptotic distribution- $\chi^{2}(27)$ (solid line), can. cor. (dashed), CD-West (dotted), LDU-West (dashed-dotted), rk-West (plusses), rk-White (stars), rk-NW (triangles).

the GMM estimates of $\gamma$ in Jagannathan and Wang (1996, Table IIC): $\gamma_{c}=2.26$ (s.e. $\left.=0.35\right)$, $\gamma_{v w}=1.81$ (s.e. $\left.=1.44\right), \gamma_{\text {int }}=-65.72($ s.e. $=21.2)$ and $\gamma_{l a b o r}=-97.72$ (s.e. $\left.=33.2\right)$. As the scale of the factors in $f_{i}$ is about the same, very large values of parameters and standard errors may indicate that the parameters are badly identified. The value of the rank statistics further indicates the issue of identification of the parameters. This also holds for most of the other specifications of the SDF in Table II of Jagannathan and Wang (1996).

\subsection{Power Comparison}

We compare the power of the different rank statistics using the same data generating process that we employed for the empirical distribution functions in Figure 1. Hence, we generate data from the model

$$
r_{i}=\Pi\left(\lambda^{*}\right) f_{i}+\varepsilon_{i}, \quad i=1, \ldots, N
$$

where $\varepsilon_{i}$ are moving average disturbances of order 1 with normal distributed innovations and

$$
\Pi\left(\lambda^{*}\right)=\tilde{\Pi}_{1}+\lambda^{*}\left(\hat{\Pi}-\tilde{\Pi}_{1}\right),
$$

with $\hat{\Pi}$ the least squares estimate of $\Pi$ for the Jagannathan and Wang (1996) data and $\tilde{\Pi}_{1}$ is the canonical correlation estimate of $\Pi$ when it has rank one. Figure 2 shows the power curves of the different statistics that test for a rank one value of $\Pi$ with $5 \%$ asymptotic significance for various values of $\lambda^{*}$. Figure 2 shows, just like Figure 1, that the rank statistics which involve the West or White covariance matrix estimators are approximately size correct. The canonical correlation rank statistic and the rank statistic that uses the Newey-West covariance matrix estimator are size distorted. The power of the rank statistics at other values of $\lambda^{*}$ behaves in a similar way, that is, the power curves of the rank statistics that use the West and White 


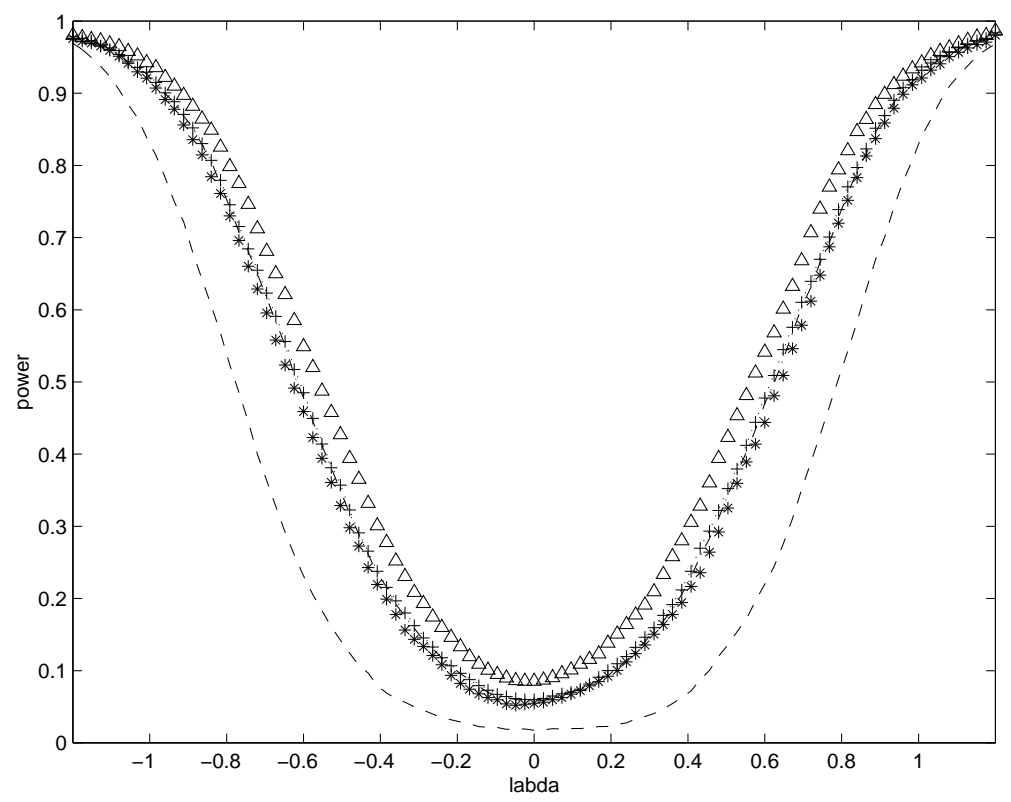

Figure 2: Power curves of rank statistics that test for rank one with $5 \%$ asymptotic significance: can. cor. (dashed), CD-West (dotted), LDU-West (dashed-dotted), rk-West (plusses), rkWhite (stars), rk-NW (triangles).

covariance matrix estimators are very similar and the power curves of the canonical correlation rank statistic and the rank statistic that involves the Newey-West covariance matrix estimator lie consistently below or above these.

\section{Concluding Remarks}

To overcome some of the deficiencies of statistics that test the rank of a matrix, we propose a novel one. The rank statistic requires a consistent estimator of the unrestricted matrix, which does not need to have a Kronecker covariance matrix. This allows one, for example, to use HACC estimators for the covariance matrix. The unrestricted matrix is decomposed using a singular value decomposition. The rank statistic is a quadratic form of an orthogonal transformation of the smallest singular values with the inverse of the respective covariance matrix. If the limiting distribution of the estimator of the unrestricted matrix is normal, the proposed rank statistic has a standard $\chi^{2}$ limiting distribution. The rank statistic simplifies to the canonical correlation rank statistic if the covariance matrix of the unrestricted matrix has a Kronecker covariance matrix. In case of non-stationary cointegrated variables, the limiting distribution of the rank statistic equals the limiting distribution of the Johansen trace statistic.

In many econometric models, the rank of a matrix governs the identification of the parameters. The limiting distribution of estimators of these parameters are only valid if this matrix has full rank. For example, in order to obtain the limiting distributions of GMM estimators, it is assumed that a matrix of derivatives has full rank, see for example Hansen (1982) and Newey and McFadden (1994). In such cases, rank statistics can be used to test for the identification of the parameters. We use rank statistic to test for the identification of the parameters in the SDF model of Jagannathan and Wang (1996). We find that lower rank values of the identifying matrix can not be rejected. The parameters in the SDF model of Jagannathan and 
Wang are therefore badly identified. This has consequences for their empirical results and the resulting conclusions.

\section{Appendix}

Proof of Theorem 1. Under $\mathrm{H}_{0}, \Theta=A_{q} B_{q}$, and Assumption 1,

$$
\|\hat{\Theta}-\Theta\| \underset{p}{\rightarrow} 0
$$

where \|\| is a distance function. We pre-multiply this expression by $\left(A_{q} \vdots A_{q, \perp}\right)^{\prime}$ and postmultiply it by $\left(B_{q}^{\prime} \vdots B_{q, \perp}^{\prime}\right)$ to obtain

$$
\left\|\left(\begin{array}{cc}
A_{q}^{\prime}\left(\hat{\Theta}-A_{q} B_{q}\right) B_{q}^{\prime} & A_{q}^{\prime} \hat{\Theta} B_{q, \perp}^{\prime} \\
A_{q, \perp}^{\prime} \hat{\Theta} B_{q}^{\prime} & A_{q, \perp}^{\prime} \hat{\Theta} B_{q, \perp}^{\prime}
\end{array}\right)\right\| \underset{p}{\rightarrow} 0 .
$$

It results from the SVD that $\hat{\Theta}=\hat{A}_{q} \hat{B}_{q}+\hat{A}_{q, \perp} \hat{\Lambda}_{q} \hat{B}_{q, \perp}$ and so

$$
\left\|\left(\begin{array}{cc}
A_{q}^{\prime}\left(\hat{A}_{q} \hat{B}_{q}-A_{q} B_{q}+\hat{A}_{q, \perp} \hat{\Lambda}_{q} \hat{B}_{q, \perp}\right) B_{q}^{\prime} & A_{q}^{\prime}\left(\hat{A}_{q} \hat{B}_{q}+\hat{A}_{q, \perp} \hat{\Lambda}_{q} \hat{B}_{q, \perp}\right) B_{q, \perp}^{\prime} \\
A_{q, \perp}^{\prime}\left(\hat{A}_{q} \hat{B}_{q}+\hat{A}_{q, \perp} \hat{\Lambda}_{q} \hat{B}_{q, \perp}\right) B_{q}^{\prime} & A_{q, \perp}^{\prime}\left(\hat{A}_{q} \hat{B}_{q}+\hat{A}_{q, \perp} \hat{\Lambda}_{q} \hat{B}_{q, \perp}\right) B_{q, \perp}^{\prime}
\end{array}\right)\right\| \underset{p}{\longrightarrow} 0 .
$$

Since the $q$ largest singular values of $\hat{\Theta}$ are contained in $\hat{A}_{q} \hat{B}_{q}, \hat{\Lambda}_{q}$ is an orthogonal transformation of the smallest singular values and $\hat{A}_{q, \perp}^{\prime} \hat{A}_{q, \perp}=I_{k-q}, \hat{B}_{q, \perp} \hat{B}_{q, \perp}^{\prime}=I_{m-q}$, the above expression implies that $\hat{A}_{q} \hat{B}_{q} \underset{p}{\rightarrow} A_{q} B_{q}, \hat{B}_{q} B_{q, \perp}^{\prime} \underset{p}{\longrightarrow} 0, A_{q, \perp}^{\prime} \hat{A}_{q} \underset{p}{\rightarrow} 0$ and $\hat{\Lambda}_{q} \underset{p}{\rightarrow} 0$. Further usage of these results and Assumption 1 gives

$$
\begin{gathered}
\sqrt{N} \operatorname{vec}\left(\hat{\Theta}-A_{q} B_{q}\right) \rightarrow \psi_{\theta} \Leftrightarrow \\
\sqrt{N} \operatorname{vec}\left(\hat{A}_{q} \hat{B}_{q}+\hat{A}_{q, \perp} \hat{\Lambda}_{q} \hat{B}_{q, \perp}-A_{q} B_{q}\right) \rightarrow \psi_{\theta} \Leftrightarrow \\
\sqrt{N} \operatorname{vec}\left(\hat{A}_{q} \hat{B}_{q}-A_{q} B_{q}\right)+\sqrt{N} \operatorname{vec}\left(\hat{A}_{q, \perp} \hat{\Lambda}_{q} \hat{B}_{q, \perp}\right) \rightarrow \psi_{\theta} .
\end{gathered}
$$

This implies that $\hat{A}_{q} \hat{B}_{q}$ is a root- $N$ consistent estimator of $A_{q} B_{q}$. Using the normalization imposed on either $\hat{A}_{q}$ or $\hat{B}_{q}$, we can show the root- $N$ consistency of the estimators involved in $\hat{A}_{q} \hat{B}_{q}$. For example, in case that $\hat{B}$ is normalized as $\hat{B}=\left[I_{q} \vdots \hat{B}_{q, 2}\right]$ with $\hat{B}_{q, 2}$ a $q \times(m-q)$ matrix,

$$
\begin{aligned}
\hat{A}_{q} \hat{B}_{q}-A_{q} B_{q} & =\left[\hat{A}_{q}-A_{q} \vdots \hat{A}_{q} \hat{B}_{q, 2}-A_{q} B_{q, 2}\right] \\
& =\left[\hat{A}_{q}-A_{q} \vdots A_{q}\left(\hat{B}_{q, 2}-B_{q, 2}\right)+\left(\hat{A}_{q}-A_{q}\right) \hat{B}_{q, 2}\right] \\
& =\left(\hat{A}_{q}-A_{q}\right)\left[I_{q} \vdots \hat{B}_{q, 2}\right]+A_{q}\left(\hat{B}_{q, 2}-B_{q, 2}\right)\left[0 \vdots I_{q}\right] .
\end{aligned}
$$

Since $\hat{A}_{q} \hat{B}_{q}$ is a root- $N$ consistent estimator of $A_{q} B_{q}, \hat{A}_{q}$ and $\hat{B}_{q, 2}$ are root- $N$ consistent estimators of $A_{q}$ and $B_{q, 2}$, respectively. Furthermore, by pre-multiplying the above expression by either $I_{k}$ or $\left(A_{q}^{\prime} A_{q}\right)^{-1} A_{q}^{\prime}$ and post-multiplying by either $B_{q}^{\prime}\left(B_{q} B_{q}^{\prime}\right)^{-1}$ or $\left[0 \vdots I_{q}\right]^{\prime}$, we obtain that

$$
\begin{gathered}
\sqrt{N} \operatorname{vec}\left(\hat{A}_{q}-A_{q}\right) \underset{d}{\rightarrow} \psi_{A}, \\
\sqrt{N} \operatorname{vec}\left(\hat{B}_{q, 2}-B_{q, 2}\right) \underset{d}{\rightarrow} \psi_{B}
\end{gathered}
$$


with $\psi_{A} \sim N\left(0, W_{A}\right), \psi_{B} \sim N\left(0, W_{B}\right), W_{A}=\left(\left(B_{q} B_{q}^{\prime}\right)^{-1} B_{q} \otimes I_{k}\right) W\left(\left(B_{q} B_{q}^{\prime}\right)^{-1} B_{q} \otimes I_{k}\right)^{\prime}$, and $\left.W_{B}=\left(\left[0 \vdots I_{q}\right] \otimes\left(A_{q}^{\prime} A_{q}\right)^{-1} A_{q}^{\prime}\right) W\left(\left[0 \vdots I_{q}\right]\right) \otimes\left(A_{q}^{\prime} A_{q}\right)^{-1} A_{q}^{\prime}\right)^{\prime}$.

If $\hat{A}_{q}$ and $\hat{B}_{q}$ are root- $N$ consistent estimators of $A_{q}$ and $B_{q}, \hat{A}_{q, \perp}$ and $\hat{B}_{q, \perp}$ are also root- $N$ consistent estimators of $A_{q, \perp}$ and $B_{q, \perp}$, respectively. This follows directly from

$$
\begin{aligned}
\hat{A}_{q}^{\prime} \hat{A}_{q, \perp} & \equiv 0 \Leftrightarrow \\
A_{q}^{\prime}\left(\hat{A}_{q, \perp}-A_{q, \perp}\right)+\left(\hat{A}_{q}-A_{q}\right)^{\prime} \hat{A}_{q, \perp} & \equiv 0,
\end{aligned}
$$

where we have used that $A_{q}^{\prime} A_{q, \perp} \equiv 0$. The fact that $\hat{A}_{q}$ is a root- $N$ consistent estimator of $A_{q}$ now implies that $\hat{A}_{q, \perp}$ is a root- $N$ consistent estimator of $A_{q, \perp}$. The same result can be obtained for $\hat{B}_{q, \perp}$.

The limiting behavior of $\hat{\lambda}=\operatorname{vec}(\hat{\Lambda})$, with $\hat{\Lambda}=\hat{A}_{q, \perp}^{\prime} \hat{\Theta} \hat{B}_{q, \perp}^{\prime}$, then results from Assumption 1 and the root- $N$ consistency of $\hat{A}_{q, \perp}$ and $\hat{B}_{q, \perp}$,

$$
\sqrt{N} \hat{\lambda}_{q}^{\rightarrow} \underset{d}{\rightarrow} \psi_{\lambda}
$$

with $\psi_{\lambda} \sim N\left(0, \Omega_{q}\right), \Omega_{q}=\left(B_{q, \perp} \otimes A_{q, \perp}^{\prime}\right) W\left(B_{q, \perp} \otimes A_{q, \perp}^{\prime}\right)$.

Proof of Proposition 1. When $V=\left(\left(F^{\prime} F\right)^{-1} \otimes\left(G^{\prime} G\right)^{-1}\right)$,

$$
W=\left(F\left(F^{\prime} F\right)^{-1} F^{\prime} \otimes G\left(G^{\prime} G\right)^{-1} G^{\prime}\right)=I_{k m} .
$$

As a consequence,

$$
\begin{aligned}
\Omega_{q} & =\left(B_{q, \perp} \otimes A_{q, \perp}^{\prime}\right) W\left(B_{q, \perp}^{\prime} \otimes A_{q, \perp}\right)^{\prime} \\
& =\left(B_{q, \perp} \otimes A_{q, \perp}^{\prime}\right)\left(B_{q, \perp}^{\prime} \otimes A_{q, \perp}\right) \\
& =I_{(k-q)(m-q)}
\end{aligned}
$$

and

$$
\begin{aligned}
\hat{\lambda}^{\prime} \Omega_{q}^{-1} \hat{\lambda} & =\operatorname{vec}(\hat{\Lambda})^{\prime} \operatorname{vec}(\hat{\Lambda}) \\
& =\operatorname{vec}\left(\hat{A}_{q, \perp}^{\prime} \hat{\Theta} \hat{B}_{q, \perp}^{\prime}\right)^{\prime} \operatorname{vec}\left(\hat{A}_{q, \perp}^{\prime} \hat{\Theta} \hat{B}_{q, \perp}^{\prime}\right) \\
& =\operatorname{tr}\left(\hat{B}_{q, \perp} \hat{\Theta}^{\prime} \hat{A}_{q, \perp} \hat{A}_{q, \perp}^{\prime} \hat{\Theta} \hat{B}_{q, \perp}^{\prime}\right) .
\end{aligned}
$$

If we write for notational convenience $U=\left[U_{1} \vdots U_{2}\right]$ with $U_{1}$ a $k \times q$ and $U_{2}$ a $k \times(k-q)$ matrix and $V=\left[V_{1} \vdots V_{2}\right]$ with $V_{1}$ a $m \times q$ and $V_{2}$ a $m \times(m-q)$ matrix, the expressions in (12) simplify to $A_{q, \perp}=U_{2} U_{22}^{-1}\left(U_{22} U_{22}^{\prime}\right)^{\frac{1}{2}}$ and $B_{q, \perp}=\left(V_{22} V_{22}^{\prime}\right)^{\frac{1}{2}} V_{22}^{\prime-1} V_{2}^{\prime}$. Substituting these values in the above expression and using the SVD expression of $\hat{\Theta}\left(=U S V^{\prime}\right)$, we obtain

$$
\begin{aligned}
\hat{\lambda}^{\prime} \Omega_{q}^{-1} \hat{\lambda} & =\operatorname{tr}\left[\left(V_{22} V_{22}^{\prime}\right) V_{22}^{\prime-1} V_{2}^{\prime} \hat{\Theta}^{\prime} U_{2} U_{22}^{-1}\left(U_{22} U_{22}^{\prime}\right) U_{22}^{-1 \prime} U_{2}^{\prime} \hat{\Theta} V_{2} V_{22}^{-1}\right] \\
& =\operatorname{tr}\left[V_{2}^{\prime} V S U^{\prime} U_{2} U_{2}^{\prime} U S V^{\prime} V_{2}\right] \\
& =\operatorname{tr}\left[V_{2}^{\prime} V_{2} S_{2} U_{2}^{\prime} U_{2} S_{2} V_{2}^{\prime} V_{2}\right] \\
& =\operatorname{tr}\left[S_{2}^{\prime} S_{2}\right]=\operatorname{tr}\left[S_{2} S_{2}^{\prime}\right]
\end{aligned}
$$

where we have used that $U_{1}^{\prime} U_{2}=0, V_{1}^{\prime} V_{2}=0, U_{2}^{\prime} U_{2}=I_{k-q}, V_{2}^{\prime} V_{2}=I_{m-q} . S_{2}$ is a $(k-q) \times(m-q)$ dimensional rectangular matrix. When $k>m$ it contains the square roots of the $m-q$ smallest 
eigenvalues of $\hat{\Theta}^{\prime} \hat{\Theta}$. When $m>q$ it contains the square roots of the $k-q$ smallest eigenvalues of $\hat{\Theta} \hat{\Theta}^{\prime}$ on its main diagonal. As

$$
\begin{aligned}
\hat{\Theta}^{\prime} \hat{\Theta} & =V S^{\prime} U^{\prime} U S V^{\prime} \\
& =V S^{\prime} S V^{\prime} \\
& =\left[V_{1} \vdots V_{2}\right]\left(\begin{array}{cc}
S_{1}^{\prime} S_{1} & 0 \\
0 & S_{2}^{\prime} S_{2}
\end{array}\right)\left[V_{1} \vdots V_{2}\right]^{\prime}, \\
\hat{\Theta} \hat{\Theta}^{\prime} & =U S V^{\prime} V S^{\prime} U^{\prime} \\
& =\left[U_{1} \vdots U_{2}\right]\left(\begin{array}{cc}
S_{1} S_{1}^{\prime} & 0 \\
0 & S_{2} S_{2}^{\prime}
\end{array}\right)\left[U_{1} \vdots U_{2}\right]^{\prime},
\end{aligned}
$$

it follows that $\operatorname{tr}\left(S_{2}^{\prime} S_{2}\right)$ is equal to the sum of the smallest $m-q$ eigenvalues of $\hat{\Theta}^{\prime} \hat{\Theta}$ and $\operatorname{tr}\left(S_{2} S_{2}^{\prime}\right)$ is equal to the sum of the smallest $k-q$ eigenvalues of $\hat{\Theta} \hat{\Theta}^{\prime}$. The eigenvalues of $\hat{\Theta}^{\prime} \hat{\Theta}$ and $\hat{\Theta} \hat{\Theta}^{\prime}$ correspond with the roots of the polynomial equations $\left|\mu \hat{F}^{\prime} \hat{F}-\hat{\Pi} \hat{G}^{\prime} \hat{G} \hat{\Pi}\right|=0$ and $\left|\mu \hat{G}^{\prime} \hat{G}-\hat{\Pi} \hat{F} \hat{F}^{\prime} \hat{\Pi}^{\prime}\right|=0$ respectively. This shows the relationship with the canonical correlations.

Proof of Theorem 2. When $\Pi=A_{q} B_{q}$ and $A_{q, \perp}^{\prime} B_{q, \perp}^{\prime}$ is non-singular, the Representation Theorem for the VAR(1) (31), see for example Johansen (1991), implies that

$$
y_{i}=B_{q, \perp}^{\prime}\left(A_{q, \perp}^{\prime} B_{q, \perp}^{\prime}\right)^{-1} A_{q, \perp}^{\prime} \sum_{j=1}^{i} \varepsilon_{j}+\xi_{i},
$$

where $\xi_{i}$ is a stationary error term and $\varepsilon_{j}=\Delta y_{j}-A_{q} B_{q} y_{j-1}$. In case of white noise disturbances $\varepsilon_{i}$ with constant covariance matrix $\Sigma$,

$$
\frac{1}{\sqrt{N \tau}} y_{\tau N} \underset{d}{\rightarrow} B_{q, \perp}^{\prime}\left(A_{q, \perp}^{\prime} B_{q, \perp}^{\prime}\right)^{-1} \Sigma_{q}^{\frac{1}{2}} B_{k-q}(\tau), \quad 0<\tau<1
$$

with $\Sigma_{q}=A_{q, \perp}^{\prime} \Sigma A_{q, \perp}$ and $B_{k-q}(\tau)$ is a $(k-q)$-dimensional Brownian motion defined on the unit interval with identity covariance matrix at time $\tau$.

From the first order condition for the least squares estimator, we obtain that

$$
\begin{aligned}
\sum_{i=1}^{N}\left(\Delta y_{i}-\hat{\Pi} y_{i-1}\right) y_{i-1}^{\prime} & =0 \Leftrightarrow \\
\sum_{i=1}^{N}\left(\Delta y_{i}-\hat{A}_{q} \hat{B}_{q} y_{i-1}-\hat{A}_{q, \perp} \hat{\Lambda}_{q} \hat{B}_{q, \perp} y_{i-1}\right) y_{i-1}^{\prime} & =0 \Leftrightarrow \\
\sum_{i=1}^{N}\left(\hat{\varepsilon}_{i}-\hat{A}_{q, \perp} \hat{\Lambda}_{q} \hat{B}_{q, \perp} y_{i-1}\right) y_{i-1}^{\prime} & =0 \Leftrightarrow \\
\sum_{i=1}^{N} \hat{\varepsilon}_{i} y_{i-1}^{\prime} & =\sum_{i=1}^{N} \hat{A}_{q, \perp} \hat{\Lambda}_{q} \hat{B}_{q, \perp} y_{i-1} y_{i-1}^{\prime} .
\end{aligned}
$$

where $\hat{\varepsilon}_{i}=\Delta y_{i}-\hat{A}_{q} \hat{B}_{q} y_{i-1}$. Since $I_{k}=P_{\hat{A}_{q}}+P_{\hat{A}_{q, \perp}}$, it follows that

$$
\sum_{i=1}^{N} \hat{\varepsilon}_{i} y_{i-1}^{\prime}=\hat{A}_{q, \perp} \hat{A}_{q, \perp}^{\prime} \sum_{i=1}^{N} \hat{\varepsilon}_{i} y_{i-1}^{\prime},
$$

where we use that $\hat{A}_{q, \perp}^{\prime} \hat{A}_{q, \perp}=I_{k-q}$. We can express $\hat{\Lambda}_{q}$ as

$$
\hat{\Lambda}_{q}=\hat{A}_{q, \perp}^{\prime}\left[\sum_{i=1}^{N} \hat{\varepsilon}_{i} y_{i-1}^{\prime}\right]\left[\sum_{i=1}^{N} y_{i-1} y_{i-1}^{\prime}\right]^{-1} \hat{B}_{q, \perp}^{\prime} .
$$

To derive the limiting behavior of the rank statistic, we first consider the limiting behavior of $\sum_{i=1}^{N} y_{i-1} \hat{\varepsilon}_{i}^{\prime}$. We specify $\hat{\varepsilon}_{i}$ as $\sum^{\frac{1}{2}} u_{i}$ with $u_{i}$ a $k \times 1$ random vector with an identity covariance matrix. When we use that $\hat{A}_{q, \perp}$ is a consistent estimator of $A_{q, \perp}$, we obtain that

$$
\frac{1}{N} \sum_{i=1}^{N} \hat{\varepsilon}_{i} y_{i-1}^{\prime} \underset{d}{\rightarrow} A_{q, \perp} \Sigma_{q}^{\frac{1}{2}}\left(\int_{0}^{1} B_{k-q}(t) d B_{k-q}(t)^{\prime}\right)^{\prime} \Sigma_{q}^{\frac{1}{2}}\left(A_{q, \perp}^{\prime} B_{q, \perp}^{\prime}\right)^{-1 \prime} B_{q, \perp},
$$


where we used that $\sum_{i=1}^{N} \hat{\varepsilon}_{i} y_{i-1}^{\prime}=\hat{A}_{q, \perp} \hat{A}_{q, \perp}^{\prime} \sum_{i=1}^{N} \hat{\varepsilon}_{i} y_{i-1}^{\prime}$ and $\Sigma_{q}=A_{q, \perp}^{\prime} \Sigma A_{q, \perp}$. The limiting behavior of second term in the expression of $\hat{\Lambda}_{q}, \frac{1}{N^{2}} \sum_{i=1}^{N} y_{i-1} y_{i-1}^{\prime}$, is given by

$$
\frac{1}{N^{2}} \sum_{i=1}^{N} y_{i-1} y_{i-1}^{\prime} \underset{d}{\longrightarrow} B_{q, \perp}^{\prime}\left(A_{q, \perp}^{\prime} B_{q, \perp}^{\prime}\right)^{-1} \sum_{q}^{\frac{1}{2}}\left(\int_{0}^{1} B_{k-q}(t) B_{k-q}(t)^{\prime} d t\right) \Sigma_{q}^{\frac{1}{2}}\left(A_{q, \perp}^{\prime} B_{q, \perp}^{\prime}\right)^{-1 \prime} B_{q, \perp}
$$

and

$$
\left(\frac{1}{N^{2}} \sum_{i=1}^{N} y_{i-1} y_{i-1}^{\prime}\right)^{-1} \underset{d}{\rightarrow} A_{q, \perp} \Sigma_{q}^{-\frac{1}{2}}\left(\int_{0}^{1} B_{k-q}(t) B_{k-q}(t)^{\prime} d t\right)^{-1} \Sigma_{q}^{-\frac{1}{2} \prime} A_{q, \perp}^{\prime}
$$

The limiting behavior of $\hat{\Lambda}_{q}$ is therefore

$$
N \hat{\Lambda}_{q} \underset{d}{\rightarrow} \Sigma_{q}^{\frac{1}{2}}\left(\int_{0}^{1} B_{k-q}(t) d B_{k-q}(t)^{\prime}\right)^{\prime}\left(\int_{0}^{1} B_{k-q}(t) B_{k-q}(t)^{\prime} d t\right)^{-1} \Sigma_{q}^{-\frac{1}{2} \prime}\left(B_{q, \perp} A_{q, \perp}\right)^{\prime}
$$

To estimate the covariance matrix of $\hat{\pi}=\operatorname{vec}(\hat{\Pi})$, we use

$$
\hat{W}=\left(\left(\frac{1}{N} \sum_{i=1}^{N} y_{i-1} y_{i-1}^{\prime}\right)^{-1} \otimes \frac{1}{N} \sum_{i=1}^{N} \tilde{\varepsilon}_{i} \tilde{\varepsilon}_{i}^{\prime}\right)
$$

where $\tilde{\varepsilon}_{i}=\Delta y_{i}-\hat{\Pi} y_{i-1}$ and hence the estimator of the covariance matrix of $\hat{\lambda}_{q}=\operatorname{vec}\left(\hat{\Lambda}_{q}\right)$ is

$$
\hat{\Omega}_{q}=\left(\hat{B}_{q, \perp} \otimes \hat{A}_{q, \perp}^{\prime}\right) \hat{W}\left(\hat{B}_{q, \perp} \otimes \hat{A}_{q, \perp}^{\prime}\right)^{\prime}
$$

The limiting behavior of $\hat{\Omega}_{q}$ corresponds with

$$
\frac{1}{N} \hat{\Omega}_{q} \underset{d}{\rightarrow}\left(B_{q, \perp} A_{q, \perp} \Sigma_{q}^{-\frac{1}{2}}\left(\int_{0}^{1} B_{k-q}(t) B_{k-q}(t)^{\prime} d t\right)^{-1} \Sigma_{q}^{-\frac{1}{2} \prime} A_{q, \perp}^{\prime} B_{q, \perp}^{\prime} \otimes \Sigma_{q}\right)
$$

and of its inverse

$$
\left(\frac{1}{N} \hat{\Omega}_{q}\right)^{-1} \underset{d}{\rightarrow}\left(\left(A_{q, \perp}^{\prime} B_{q, \perp}^{\prime}\right)^{-1} \Sigma_{q}^{\frac{1}{2}}\left(\int_{0}^{1} B_{k-q}(t) B_{k-q}(t)^{\prime} d t\right) \Sigma_{q}^{\frac{1}{2}}\left(A_{q, \perp}^{\prime} B_{q, \perp}^{\prime}\right)^{-1 \prime} \otimes \Sigma_{q}^{-1}\right) .
$$

From this we obtain the limiting behavior of the $\operatorname{rank}$ statistic $\operatorname{rk}(q)=\frac{1}{N} \hat{\lambda}_{q}^{\prime} \hat{\Omega}_{q}^{-1} \hat{\lambda}_{q}$,

$$
\operatorname{rk}(q) \underset{d}{\rightarrow} \operatorname{tr}\left[\left(\int_{0}^{1} B_{k-q}(t) d B_{k-q}(t)^{\prime}\right)^{\prime}\left(\int_{0}^{1} B_{k-q}(t) B_{k-q}(t)^{\prime} d t\right)^{-1}\left(\int_{0}^{1} B_{k-q}(t) d B_{k-q}(t)^{\prime}\right)\right]
$$




\section{References}

[1] Anderson, T.W. Estimating linear restrictions on regression coefficients for multivariate normal distributions. Annals of Mathematical Statistics, 22:327-351, 1951.

[2] Andrews, D.W.K. Heteroskedasticity and Autocorrelation Consistent Covariance Matrix Estimation. Econometrica, 59:817-858, 1991.

[3] Campbell, J.Y., A.L. Lo and A.C. MacKinlay. The Econometrics of Financial Markets. Princeton University Press, 1997.

[4] Cragg, J.C. and S.G. Donald. On the Asymptotic Properties of LDU-Based Tests of the Rank of a Matrix. Journal of the American Statistical Association, 91:1301-1309, 1996.

[5] Cragg, J.C. and S.G. Donald. Inferring the rank of a matrix. Journal of Econometrics, 76:223-250, 1997.

[6] Engle, R.F. and C.W.J. Granger. Co-integration and error correction : Representation, estimation and testing. Econometrica, 55:251-276, 1987.

[7] Gill, L. and A. Lewbel. Testing the Rank and Definiteness of Estimated Matrices With Applications to Factor, State-Space and ARMA Models. Journal of the American Statistical Assocation, 87:766-776, 1992.

[8] Golub, G.H. and C.F. van Loan. Matrix Computations. The John Hopkins University Press (Baltimore), 1989.

[9] Hansen, L.P. Large Sample Properties of Generalized Method Moments Estimators. Econometrica, 50:1029-1054, 1982.

[10] Jagannathan, R. and Z. Wang. The Conditional CAPM and the Cross-Section of Expected Returns. Journal of Finance, 51:3-53, 1996.

[11] Jagannathan, R., G. Skoulakis and Z. Wang. Generalized Method of Moments: Applications in Finance. Journal of Business and Economic Statistics, 20-4:470-481, 2002.

[12] Johansen, S. Estimation and hypothesis testing of cointegration vectors in Gaussian vector autoregressive models. Econometrica, 59:1551-1580, 1991.

[13] Johansen, S. Likelihood-Based Inference in Cointegrated Vector Autoregressive Models. Oxford University Press, Oxford, 1995.

[14] Kleibergen, F. and H.K. van Dijk. Direct Cointegration Testing in Error Correction Models. Journal of Econometrics, 63:61-103, (1994).

[15] Lewbel, A. The Rank of Demand Systems: Theory and Nonparametric Estimation. Econometrica, 59:711-730, 1991.

[16] Newey, W.K. and D. McFadden. Large Sample Estimation and Hypothesis Testing. In R. Engle and D. McFadden, editor, Handbook of Econometrics, Volume 4, chapter 36, pages 2113-2148. Elsevier Science B.V., 1994. 
[17] Newey, W.K. and K.D. West. A Simple Positive Semi-Definite Heteroskedasticity and Autocorrelation Consistent Covariance Matrix. Econometrica, 55:703-708, 1987.

[18] Phillips, P.C.B. and V. Solo. Asymptotics for Linear Processes. Annals of Statistics, 20:971-1001, 1992.

[19] Robin, J.-M. and R.J. Smith. Tests of Rank. Econometric Theory, 16:151-175, 2000.

[20] West, K.D. Another heteroscedasticity- and autocorrelation-consistent covariance matrix estimator. Journal of Econometrics, 76:171-191, 1997.

[21] White, H. A Heteroskedasticity-Consistent Covariance Matrix Estimator and a Direct Test for Heteroscedasticity. Econometrica, 48:817-838, 1980.

[22] Wright, J.H. Detecting Lack of Identification in GMM. Econometric Theory, 2003, 19: 322-330, 2003. 